\title{
Family Tensions and Information Privacy: A Barrier to Diffusion of Proximity Tracing Applications?
}

\author{
France Bélanger*, Robert E. Crossler**, Katherine R. Allen*, \\ Jessica Resor*, Heather A. Kissel*, Travis Finch* \\ *Virginia Tech \\ ** Washington State University \\ belanger@vt.edu, rob.crossler@wsu.edu, kallen@vt.edu, imresor@vt.edu, hkissel2@,vt.edu, \\ tfinch21@vt.edu
}

\begin{abstract}
Technology played a central role during the pandemic for communications and services. It was also touted as a potential solution to control the spread of COVID-19 via proximity tracing applications, also known as contact tracing (CT) apps worldwide. In non-mandated settings, however, these apps did not attain popularity. Privacy concerns were highlighted as one reason. We explored how family perceptions of CT apps can affect the family's use of such apps. We surveyed parent-teen dyads twice over a 5-month period. We analyzed parent-teen perceptions of each other's intentions and use of CT apps at time 1 and 2, exploring changes over time. Parents' use intentions were influenced by their and their teens' perceptions of the benefits but not privacy concerns. Teen intentions were influenced by their own perceptions of benefits, not their parent's, and their parent's concerns for the family. Intentions always influenced usage, including intentions at time 1 influencing use at time 2, demonstrating a longitudinal effect of intentions on usage existed for parents and teens.
\end{abstract}

\section{Introduction}

When the COVID-19 pandemic threw the world into a global health crisis and led to the largest economic downturn since the great depression, people, organizations, and governments turned to technology for key aspects of life, including obtaining food and health care, work and school, communication, and entertainment. Technology was also viewed as a possible 'solution' to mitigate the spread of the disease via the use of proximity tracing apps, also known as contract tracing (CT) apps. Utilizing CT apps would allow for quick notification for people who have been in contact with someone with a positive COVID-19 result. To be successful, researchers suggested that $80 \%$ of smartphone users had to use the CT app to help keep the r-naught of COVID-19 below 1 (which is the number of people the COVID positive person spread the virus to), which would reduce its spread [1]. However, CT apps did not attain the required levels needed to be efficient, except in countries that mandated their use. For example, South Korea was regarded as an exemplar of containing the COVID-19 outbreak by implementing a mandatory, or required, centralized technological monitoring system that identified patients with extensive details and delivered warning messages to anyone who had entered the patient's proximity [2]. Meanwhile, in many Western countries such as the United States (US) and the United Kingdom (UK), diffusion of CT apps was slow to catch on. In the US, this was due to the voluntary and decentralized approach at diffusion, while the UK's privacy laws prevented their implementation [2, 3]. Nearly a year after the first US state, Virginia, launched a CT app, just over $25 \%$ of the population had used the service, as claimed by the Commonwealth of Virginia Department of Health [4]. Research has also shown that even when COVID-19 concerns were high that privacy concerns significantly reduced intentions to download a CT app [5].

While vaccines that were developed and are still being distributed (at the time this paper was submitted) have 'reduced' the need for CT apps, governments and businesses need to better understand why the diffusion of 'voluntary' CT programs were not effective because other community technologies will be needed for handling future community and societal crises. One example is the current debate about the use of technology-driven vaccine passports [6]. It is clear that citizens did not want to relinquish their information privacy to the level required for $\mathrm{CT}$ apps to work properly, with $71 \%$ of adults saying they would not 
use the app due to privacy concerns [7]. Complicating the challenges of community technology acceptance with respect to privacy, at least in the case of CT apps, is that many households are inter-generational and each family member needs to decide if they allow CT apps to run on their smartphone. Any individual decision could affect the entire household. For example, if families go to dinner together and one member has the CT app turned on, the entire family is functionally traced. At the same time, not all family members may view the relative advantages of using the CT apps similarly.

In this research, we argue that these within-family tensions on perceptions (including privacy) of CT apps affect a family's ultimate choice to use the apps, which then could have a ripple effect on broader community adoption. The research is guided by the following question: what are the barriers and enablers to within-family adoption of proximity tracing applications? To answer this question, we surveyed parent-teen dyads twice over a 5-month period during the COVID-19 pandemic. Drawing from the privacy calculus model but extending its application within dyads (instead of individuals) and over time, we examined the balance between perceived benefits and information privacy concerns in affecting intentions and actual (self-reported) use of CT apps. Our findings suggest that parents' intentions to use CT apps were influenced by their own and their teens' perceptions of the benefits of such use but not their or their teens' privacy concern for themselves or the family. Teen usage intentions were influenced by their own perceptions of the benefits of CT apps, but not their parent's. Teen intentions were also influenced by their parent's privacy concerns for the family. All intentions influenced self-reported usage. A longitudinal effect of intentions on usage existed for parents and teens.

\section{Background}

\subsection{Contact tracing as family and community technologies}

The decision to use a CT app affects not only the individual but also others who live with them. This is particularly salient in situations where families were huddled together during the pandemic with stay-athome orders and lockdowns. While some individual family members might have been more afraid of contracting COVID-19 due to health conditions or other reasons, another family member might be too concerned about their privacy to encourage use of CT apps in the family. In fact, $71 \%$ of families stated not wanting to use CT apps due to privacy reasons [7]. However, if one family member's work environment mandates the app's use, this economic concern may override privacy concerns. Alternatively, a family member may become hopeful that the app will allow them to resume a more normal life while others remain concerned about privacy. Individual usage decisions can thus be fueled by each individual's perceptions, emotions, and circumstances, but the decision can also affect other family members, possibly generating tensions between individual and family perceptions and decisions.

A family is a complex and dynamic relational structure that involves a mix of care, concern, and conflict [8]. Families can range from small dyads to complicated inter-generational family systems that interact and transact within their wider communities and macro-level environments [9]. Communication processes in families are used to express emotions and cognitions, including love, affection, problem solving, and constructive dialogue, as well as neglect, conflict, and aggression [10]. Technology, however, has changed how families manage these interactions and transactions, leading to more complex family dynamics [10-12]. In fact, many researchers have suggested that parents are often challenged and concerned that their children may know more than they do about the technologies used within the household [11, 13]. As a result of these possible intergenerational conflicts, the equilibrium in the family's relationships may be more difficult to maintain. This is particularly true for fathers, who can feel less competent when their expertise is challenged or when their children seem to be ahead of the curve with their understanding of technologies [14, 15]. Therefore, parents may not be able to impose their views or influence other family members on the use of CT apps on smartphones within the household in this technological context. Consequently, how are familylevel decisions about information privacy and the use of CT apps made?

\subsection{Privacy decisions in families}

Unfortunately, there is limited research on family decisions about the sharing of information. Families can include complex relationships where one member's privacy decisions can affect other members of the family. In this paper, we argue that privacy decisions in families involve a combination of individual and family level considerations, such as concerns for other family members. The literature, however, offers several theoretical frameworks for understanding information privacy decisions. Privacy calculus is one of the most well-established frameworks in this area [e.g., 16, 17], which we use as a theoretical foundation to explore the considerations 
each family member takes in deciding to use (and thus share information with) a CT app.

The privacy calculus suggests that individuals' privacy decisions are based on their estimates of the costs and benefits (cost-benefit tradeoff) of a decision [e.g., 16, 17]. Grounded in the developmental theory of privacy [18], the privacy calculus involves the perceptions of the decision maker on what the possible consequences of releasing information will be and whether those consequences can be managed [19]. In our study, the benefits of releasing information to CT apps could be health related (e.g., knowing to get tested or not, knowing to quarantine or not, feeling safer) or convenience related (e.g., being able to go to the gym, ability to continue working or see family members). The most studied cost component of the privacy calculus is privacy concern [e.g., 16, 17], or worrying that revealing information could have negative consequences. The balance between privacy concerns and perceived benefits should predict intentions, as suggested by the calculus, and perceived benefits is often the strongest of the two components [e.g., 20].

While the privacy calculus has been criticized for taking a mostly rational view of privacy decisions, the complex nature of families requires an initial investigation into how the perceptions of the relative costs and benefits can affect decisions in contexts where there are more complex relationships among different inter-dependent individuals. In fact, the privacy calculus, to our knowledge, has not be used to study interdependent dyads as we do in this work, nor has it been used with longitudinal data. We discuss this latter point in the next sub-section. Finally, it should be noted that the privacy calculus is typically used to "predict" willingness to share information or intention to use a technology that requires sharing of information [e.g., 16, 21, 22]. However, many researchers have called for moving beyond intentions to measure actual privacy outcomes [23, 24].

\subsection{Hypotheses development}

Given the context of families (i.e., parent-teen dyads in our study) and CT decisions, we expected that the privacy calculus would hold and the intentions to use CT apps for individuals would be higher if perceived benefits are viewed as high and privacy concerns are seen as low. However, how do these relationships play out across the dyads? Family science literature suggests that typically parents can influence their teens' perceptions and ultimate decisions since parents are the primary socialization agent for children. Parental influence is a lifelong process, as well. Parents have the most influence when children are very young, and the lessons they instill in children persist, even as children develop and are influenced by peers, other adults, media, and other social institutions. Cognitive and emotional patterns are a complex mix of nature (biology and temperament), nurture (parental and familial nurturance and learning), as well as the dialectical interaction among them [8-10, 25-27]. Teens are also under parental influence. Recent mixed methods research on the transition from adolescence to young adulthood among a sample of participants aged 19-25 found that parents were perceived as highly influential on participants' digital technology use, especially when they were children. When older adolescents become adults and live apart from their families, parental influence becomes less directly influential and is more variable [25].

Thus, parents are especially powerful sources of influence over their children, whether they are aware of the impact of their ideas, values, and behavior or not. In addition to the developmental tasks of parenting, which include meeting the physical, emotional, social, and safety needs of children, most family learning occurs under the radar, through modeling and observation, rather than through direct verbal communication. The need for formal parenting education, especially as societies are transformed by the digital revolution, and teens are preparing for jobs in the future that rely on technology and automation, is ever-increasing [27]. Recognizing this, laws exist to ensure that parents have some say in what children can do in the online environment [28].

Thus, families offer a unique and innovative environment to study information privacy because of the mix of caring, concerns, and conflicts we previously discussed [8]. While normally information privacy concerns are measured about the self [29], it is important in the context of families to also measure how one's concern for family members might affect their information disclosure to a CT app decisions. This is particularly salient in the context of the pandemic since families might include one or more members that have health conditions, or members that are required to work as essential workers, such that privacy concerns may not be the same for the self and the family. There are few studies that consider concern about the privacy of others, with the exception of studies of decisions made about posting information or pictures of others on social media [e.g., 30, 31]. This study thus adds to that literature but in the context of families.

Consistent with the theoretical foundation and information systems and family science literatures but extending the privacy calculus to a dyadic relationship of parents-teens, where privacy concerns can be for the 
self and/or the family, we expected that parent's and teen's perceptions and decisions regarding CT apps will influence each other, as stated in the hypotheses below.

- H1a: Parents' perceived benefits of using a contact taking app will be positively related to their teens' intentions to use a CT app.

- H1b: Parents' privacy concern toward CT apps will be negatively related to their teens' intentions to use a CT app.

- H1c: Parents' privacy concern for their family toward CT apps will be negatively related to their teens' intentions to use a CT app.

The traditional family science literature suggests that a positive and important component of authoritative parenting is parental firmness, which involves imposing limits and boundaries on their children's behaviors to facilitate self-regulation [26]. At the same time, recent literature suggests that in the context of technologically related decisions, children (teens) may actually influence decisions because they may know more about technology than their parents do $[11,13]$. In this new and more malleable environment, especially with today's continuous access to on-demand information, the traditional parent-child hierarchy is challenged and parental authority may not lead to the influences we would normally expect [14]. In the context of CT apps, it is therefore possible that teens - heavy users of smartphones - may be more apt to influence the family's perceptions and intentions towards usage. We therefore explored the possibility that it is the teens' perceptions that will influence parents' intentions.

- $\quad$ H2a: Teens' perceived benefits of using a CT app will be positively related to their parents' intentions to use a CT app.

- H2b: Teens' privacy concern toward CT apps will be negatively related to their parents' intentions to use a CT app.

- H2c: Teens' privacy concern for their family toward CT apps will be negatively related to their parents' intentions to use a CT app.

Finally, consistent with the prior discussion that only measuring intentions is not sufficient in the realm of information privacy decisions, we proposed that intention would lead to behaviors. The recent years have seen an increased number of studies measuring the intention-behavior link [e.g., 32], but this relationship should not be simply assumed to exist [23].

- H3: Parents' intentions to use a CT app will be positively related to their (self-reported) use of a CT app.

- H4: Teens' intentions to use a CT app will be positively related to their (self-reported) use of a CT app.

Most theories, models, and studies of information privacy focus on willingness to disclose information at one point in time, neglecting to take into account changes over time [24]. Yet, several environmental and contextual factors change over time and may influence later decisions, especially regarding child and family development. In fact, reflecting on prior information privacy decisions (such as the actual costs and benefits obtained) could influence future decisions [19] if changes are noted in the realization of the costbenefit trade-off. While we have not been able to identify longitudinal studies of cost-benefit tradeoffs and their effects on privacy intentions and/or behaviors, it is reasonable to assume that if the costs and benefits continue to be similarly perceived over time, intentions should continue to lead to privacy behaviors. In other words, if individuals continue to perceive greater benefits from the use of CT apps over their possible concerns (costs), then intentions to disclose information to and use CT apps at T1 should affect use of the apps at T2.

- H5: In the situation where the perceived costbenefit tradeoff remains the same over time, parents' intentions to use a CT app at T1 will be positively related to their (self-reported) use of a CT app at T2.

- H6: In the situation where the perceived costbenefit tradeoff remains the same over time, teens' intentions to use a CT app at T1 will be positively related to their (self-reported) use of a CT app at T2.

\section{Methodology}

For this research, we collected longitudinal data by conducting two surveys of dyads (parent-teen) over a five-month period during the COVID-19 pandemic. Subjects were recruited via Qualtrics (Qualtrics.com). 


\subsection{Participants}

For both timepoints (T1, September-October 2020; T2, February-March 2021), we recruited participants via Qualtrics panel services in different regions of the US. Compensation was provided by Qualtrics, and the exact payment to participants is unknown to the research team. We requested from the panel service (and obtained) diversity in terms of gender, race, ethnicity, education, occupation, age, and geographic locations. To participate, one parent and their teenager (13-17 years old) who lived with them had to be available to fill out the survey. The choice to survey teenagers as opposed to younger children was to ensure that (a) they had a smartphone, and (b) they had achieved the developmental capacity to make decisions about information sharing [33, p. 433]. At T1, 379 parent-teen pairs completed the survey. At T2, 304 parent-teen pairs participated. There was a $29 \%$ retention rate from $\mathrm{T} 1$ to $\mathrm{T} 2$. Thus, our sample of unique parent-teen pairs was 573, but only 110 dyads can be analyzed longitudinally. All dyads can be analyzed for within family tension hypotheses. Our sample was geographically dispersed throughout the South (39.3\%), West (21.4\%), Northeast (19.8\%), and Midwest (19.5\%) regions of the US and racially distributed similarly to that of the US. Most parents were employed full time (62.8\% T1 and $67.8 \% \mathrm{~T} 2)$ and more parents considered their employment essential at T1 $(51.9 \%)$ than at T2 (46\%). Both teen and parent samples had relatively equal gender distribution (45\% male parents at $\mathrm{T} 1$ and $50 \%$ at $\mathrm{T} 2 ; 56 \%$ male teens at $\mathrm{T} 1$ and $51 \%$ at $\mathrm{T} 2$ ). The majority of respondents were white (74\% T1 to $82 \%$ $\mathrm{T} 2)$ and the majority of parents were married (72\% T1 and $74 \% \mathrm{~T} 2$ ). There was a wide variety of educational backgrounds and income levels.

\subsection{Survey instruments}

The surveys consisted of mainly quantitative measures supplemented by open-ended qualitative questions and examined how decisions regarding usage of CT apps are negotiated within households and how to foster CT app acceptance within families. The surveys were developed using or adapting previous scales and literature from information systems and family science research fields. The survey instruments (pre and post) underwent several pretesting efforts where colleagues and personal contacts (i.e., parents and teens) went through the instrument and provided feedback, and were then pilot tested with 71 dyads of student roommates (living together) at a large public university, as well as a few parent-teen dyads.
In the main data collection, dyads took approximately 30 to 40 minutes to complete the surveys at each time point. The surveys were developed such that the intentions to use CT apps were measured within the first questions while the actual use of the CT app was included towards the end of the survey and embedded in a question containing eight preventive behaviors (e.g., wearing masks, social distancing, washing hands, disinfecting services) so that it would be less likely to be influenced by their earlier responses to the intentions questions. The surveys were also structured such that parents granted parental permission to their teen to participate, and teens assented electronically. However, neither parents nor teens could return back through the survey to see each other's answers. Attention check questions were also used throughout the survey for data quality purposes, as well as a marker variable for scale psychometric measurements. Survey items are available by contacting the first author. Parents and their teens took the initial surveys (parent section first followed by teen section) at $\mathrm{T} 1$, and then took the same survey questions in the same order at $\mathrm{T} 2$.

\subsection{Data analyses}

Prior to hypothesis tests, we cleaned the data, removing any observations that had substantial missing data points, and then assessed the scales' psychometric properties using convergent and discriminant validity, and reliability tests. Cronbach's $\alpha$ for each construct are above the recommended value of 0.70 . The range of alpha values was 0.786 for perceived benefits to 0.966 for family-related privacy concerns. Average variance extracted (AVE) for each construct exceeds 0.50 , fulfilling the requirement for convergent validity. The square root of each construct's AVE is greater than the correlations between each pair of constructs in rows and columns, demonstrating discriminant validity. All items loaded with their respective factors.

Before addressing dyadic influences, preliminary model tests were conducted to determine if the privacy calculus held for the dataset as a whole and within the two participant types (i.e., parents and teens). All structural equation models were calculated using maximum likelihood estimation in the open-source $\mathrm{R}$ package lavaan [34]. All variables were standardized prior to analysis. The initial model examined the influence of perceived privacy concerns (eight items total; four items for concern for self; four items for concern for family) and perceived benefits (11 items) on intention to use CT apps and the relationship between intention to use CT apps and actual use. The standard privacy calculus model of costs and benefits 
affecting intentions and then behaviors for the overall $\mathrm{T} 1$ dataset (comprising parents and teens) demonstrated good model fit (RMSEA $=0.06$, $\mathrm{NNFI}=0.94)$ and all paths were significant $(\mathrm{p} \leq 0.001)$. This indicates that the privacy calculus held for within person $\mathrm{T} 1$ data. In fact, multiple group analyses revealed configural and metric invariance between parents and teens, suggesting both groups interpreted the scale items similarly.

We then expanded the model described above to test for dyadic influences and conducted paired samples t-tests and calculated an additional structural equation model to examine longitudinal effects. The model's expansion to include dyadic relationships (i.e., benefits, concerns, intentions of both members of the dyad influencing each other) was based on the commonly utilized actor-partner interdependence model for analyzing distinguishable dyads [35]. The conceptual model that served as the basis for the dyadic analyses appears in Figure 1. The model was tested using the full $\mathrm{T} 1$ dataset for dyadic examinations. The $\mathrm{T} 1$ and $\mathrm{T} 2$ data for the parents and teens who completed the survey at both timepoints was used for testing the longitudinal hypotheses. Path coefficients and effect sizes were compared between the T1 and T2 models.

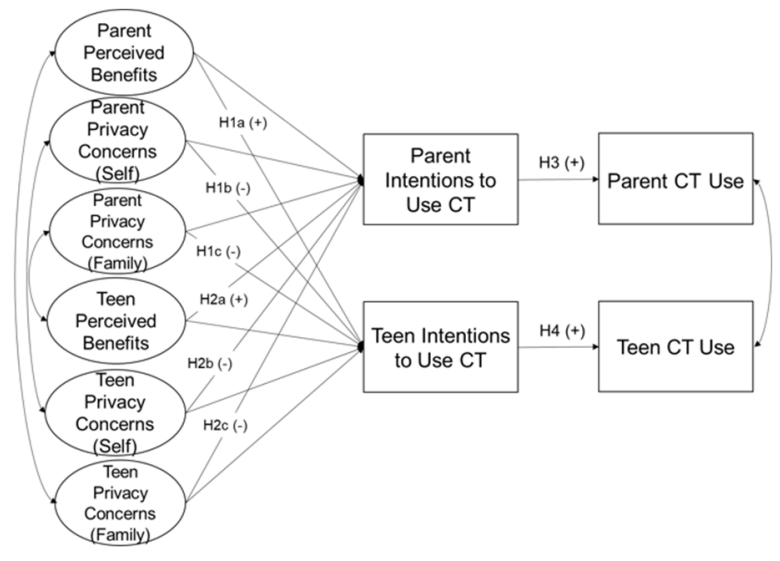

Figure 1. Conceptual model of dyadic
analyses

\section{Results}

Table 1 summarizes the standardized path values, standard errors, and $p$ values for the tests of the proposed relationships at T1. For testing T1 relationships (H1 to $\mathrm{H} 4)$, we used the complete set of parent-teen dyads. The model demonstrated good fit (RMSEA $=0.055, \mathrm{CFI}=0.92$ ). To ensure that the results were robust, we also tested the relationships with the 110 parent-teen dyads that had completed T1 and T2 data collection. The resulting patterns and significance were similar to the results for the complete dataset of dyads. The analysis of the actor and partner effects indicates that dyadic influences adjust the privacy calculus outcomes for both parents and teens.

At T1, parents' intentions to use CT apps and thus disclose information to these apps were strongly influenced by their perceptions of the benefits of such use, but that did not affect their teen's intentions to use the app (H1a). Their privacy concerns about CT apps did not affect their own intentions, but their concerns for the family did influence their teen's intentions. Thus, H1b was not supported, but H1c was.

Teen perceptions of the benefits of CT apps positively influenced their own intentions, and importantly influenced their parent's intentions (H2a). However, their privacy concerns for both themselves and their family did not affect their own intentions nor their parent's. Thus, $\mathrm{H} 2 \mathrm{~b}$ and $\mathrm{H} 2 \mathrm{c}$ were not supported. For the relationships from intentions to behaviors, we found very strong links for both parents and teens. The use of CT apps by parents and teens was significantly positively predicted by their own intentions at $\mathrm{T} 1$, supporting $\mathrm{H} 3$ and $\mathrm{H} 4$. A summary of those results are presented in Figure 2.

Table 1. Dyadic influence hypothesis testing (at Time T1)

\begin{tabular}{|l|r|r|r|}
\hline Path & \multicolumn{1}{|c|}{$\beta$} & \multicolumn{1}{|c|}{ SE } & \multicolumn{1}{|c|}{$p$} \\
\hline Predicting Parent Intentions \\
\hline Parent benefits $\rightarrow$ & 0.74 & 0.31 & $<\mathbf{0 . 0 1}$ \\
\hline Parent concern(s) $\rightarrow$ & 0.27 & 0.21 & 0.81 \\
\hline Parent concern(f) $\rightarrow$ & -0.31 & 0.20 & 0.06 \\
\hline H2a:Teen benefits $\rightarrow$ & 0.82 & 0.30 & $<\mathbf{0 . 0 1}$ \\
\hline H2b:Teen concern(s) $\rightarrow$ & 0.28 & 0.26 & 0.73 \\
\hline H2c:Teen concern(f) $\rightarrow$ & -0.27 & 0.27 & 0.15 \\
\hline Predicting Teen Intentions \\
\hline H1a:Parent benefits $\rightarrow$ & 0.30 & 0.30 & 0.16 \\
\hline H1b:Parent concern(s) $\rightarrow$ & 0.39 & 0.21 & 0.94 \\
\hline H1c:Parent concern(f) $\rightarrow$ & -0.37 & 0.20 & $\mathbf{0 . 0 3}$ \\
\hline Teen benefits $\rightarrow$ & 1.28 & 0.33 & $<\mathbf{0 . 0 1}$ \\
\hline Teen concern(s) $\rightarrow$ & -0.04 & 0.25 & 0.45 \\
\hline Teen concern(f) $\rightarrow$ & -0.02 & 0.26 & 0.48 \\
\hline Predicting use of CT Apps \\
\hline H3: Parent intent $\rightarrow$ Use & 0.37 & 0.04 & $<\mathbf{0 . 0 1}$ \\
\hline H4: Teen intent $\rightarrow$ Use & 0.38 & 0.04 & $<\mathbf{0 . 0 1}$ \\
\hline
\end{tabular}

Note: $\mathrm{f}=$ concern for family's privacy; $\mathrm{s}=$ for self 


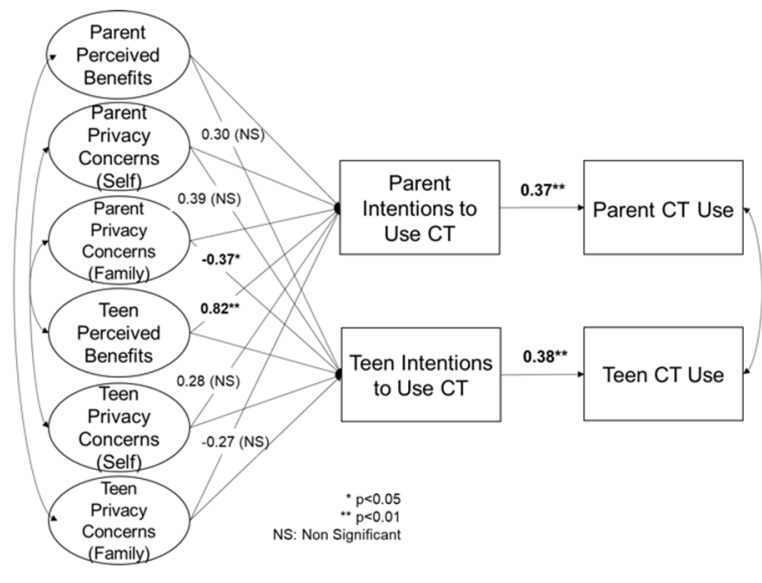

Figure 2. Results

To test for longitudinal effects (H5 and H6), we first compared summative perceived benefits and the mean of the privacy concern scales at $\mathrm{T} 1$ and $\mathrm{T} 2$ for parents and teens using paired sample t-tests. There were no significant differences in the mean number of perceived benefits at $\mathrm{T} 1$ versus $\mathrm{T} 2$ for either parents (T1: $M=2.26, \mathrm{~T} 2: M=2.20, p=.81$ ) or teens (T1: $M=2.67$, T2: $M=2.28, p=.11$ ), and there were no significant differences in their privacy concern perceptions for themselves (parents T1: $M=3.33$, parents T2: $M=3.35, p=.88$; teens T1: $M=3.43$, teens T2: $M=3.41, p=.83$ ) or their families (parents $\mathrm{T} 1$ : $M=3.46$, parents $\mathrm{T} 2: M=3.51, p=.68$; teens $\mathrm{T} 1$ : $M=3.48$, teens T2: $M=3.36, p=.23)$. Thus, there were no significant changes to the cost-benefit tradeoff perceptions.

We then conducted an actor-partner interdependence model (testing for actor effects only) using the overlapping parent-teen dyads from $\mathrm{T} 1$ and T2. The standardized path values, standard errors, and $\mathrm{p}$ values appear in Table 2. Both parent's and teen's intentions to use CT apps at $\mathrm{T} 1$ significantly predicted their own (self-reported) use of CT apps at T2. Therefore, while neither the teens nor the parents' perceptions of benefits or privacy concerns changed significantly from $\mathrm{T} 1$ to $\mathrm{T} 2$, their use of $\mathrm{CT}$ apps at $\mathrm{T} 2$ was affected by their initial intentions, supporting H5 and $\mathrm{H} 6$.

Table 2. Longitudinal results

\begin{tabular}{|l|c|c|c|}
\hline Path & $\beta$ & SE & $p$ \\
\hline Parent CT App Use (T2) & \multicolumn{4}{|l|}{} \\
\hline H5:Parent Intent (T1) $\rightarrow$ & 0.24 & 0.08 & $<\mathbf{0 . 0 1}$ \\
\hline Teen CT App Use (T2) \\
\hline H6:Teen Intent (T1) $\rightarrow$ & 0.24 & 0.08 & $<\mathbf{0 . 0 1}$ \\
\hline
\end{tabular}

\section{Discussion}

This research investigated the effects of perceptions of benefits versus privacy concerns (costs) in families on the intentions to use CT apps and the relationship between these intentions and self-reported usage at two time points. The initial tests showed that the privacy calculus model is supported again by these data, indicating its robustness into the context of CT apps. The interesting findings, however, and the goals of this research were to test these effects in dyadic relationships and over time. We thus organize our discussion around those two unique settings for this research.

Our first four hypotheses (H1ab and H2ab) were developed by drawing on family science theory and research to understand the mutual effects that parents' and teens' perceptions would have on each other's intentions to use CT apps. What we found is that parents' intentions are driven by the perceived benefits of CT apps, both their and their teens, but not concerns. The larger effects of benefits on intentions is consistent with much of the privacy calculus literature [e.g., 32].

Contrary to expectations, teens' intentions are driven by their own perceived benefits of CT apps and their parent's concern for privacy of the family. While not consistent with the overall idea of the calculus (where their own privacy concern should affect their intentions), this is consistent with family science literature where teens start to show independence from parents so that their personal perceptions of benefits start to matter more. In fact, Blackwell et al. [36] suggest that when parents communicate about technology rules, teens only hear what not to do (negative connotation), but not what to do. In the context of our study even if parents tout the benefits of CT apps, teens might still base their decisions on their own perceptions of benefits. However, if parents have also regularly discussed the importance of privacy for the family, that may also influence teens' intentions. There is an important implication for parental education and society in this finding. Parents should encourage positive technology use [27], especially for community technologies like health assessment apps or CT apps, not simply by stating the general benefits of such use, but also by ensuring that their teens clearly understand the benefits for themselves, as well as the privacy implications for their families. Alternatively, teens may be rebellious and choose to do the opposite of what their parents would like them to do. This would represent a family tension regarding choosing to make health decisions that would benefit society and demonstrating an independence that is different from what parents' desire. 
We see a tension in these results between parents and teens in considering costs and benefits of disclosing information to CT apps. The pandemic offered parents and teens the opportunity to negotiate rules around smartphone usage and privacy impacts. It is not clear, however, that such discussions were held openly. As technology continues to invade family lives, there is a need to better understand how parents can mediate their teen's use of technology (and media). For example, how do parents mediate the teens' use of other technologies like TV consumption or computing device screen times? Unfortunately, there is a social class issue that is related to parental mediation of children and teens' use of technology. Clark [37] suggests that children's rights may conflict with their parents' best intentions and in less advantaged families, the emphasis is on family rights as a whole while more advantaged families may give more space for children's rights and opinions. It seems like the pandemic has exacerbated already existing social class divides and widened inequalities [27], and this divide's effect on intentions to use proximity tracing applications is in need of further research.

One of the possible explanations for the lack of influence of parents' perceptions of benefits (and concerns) on teen's intentions could be the lack of understanding that teens have of what CT apps can really do. We have anecdotal data from a qualitative question where we asked teens and parents about why they answered their intention question the way they did. The question was optional but of those who responded, many teens indicated that they did not want to use CT apps for fear that their parents could track them. This clearly suggests that knowledge was a problem because the app cannot do that. The lack of understanding of CT programs has been named as a possible reason for the lack of successful diffusion of CT apps [38]. The question then is what is the relative role of privacy as one reason for unsuccessful CT programs [39], relative to poor coordination of rollout efforts [38] and knowledge?

Our results show that intention is a strong predictor of (self-reported) use of $\mathrm{CT}$ apps (H3 and H4). This supports established theories, such as the Theory of Planned Behavior, and the calls for more research that measures behaviors, not just intentions [e.g., 23, 40-42]. Those findings also offer some new insights. First, it shows that the results are robust across the two types of respondents (parents-teens). Second, our results also offer some insights into the role of the privacy calculus over time. Perceptions of the cost-benefit tradeoffs did not change, and both parents and teens' use of CT apps at T2 were affected by their initial intentions (supporting $\mathrm{H} 5$ and H6). This provides initial support for a longitudinal privacy calculus, but much more research is needed to further understand the effect of time on the privacy calculus and in particular teens' decisions to use technologies. What if their perceptions of teens or parents had changed? Would the link from intention to behavior be different than our findings?

Our study provides significant contributions to both information systems and family science literatures. First, it offers a theoretical understanding of how to foster CT acceptance within families and possibly other social units. This theoretical understanding should apply to other community technologies such as health assessment apps (e.g., used to access gyms during the pandemic) or the vaccination passports that are being discussed at length in today's media. The study also helps inform the literature about how decisions about usage of CT apps are negotiated within households, so that future research can examine other home technologies that impact additional family members. For example, when families acquire smart home speakers, who in the family is in charge of settings? Who has control of what is being "played" on the device?

The study also helps extend the privacy calculus literature to the dyadic context of parents and teens. Clearly, benefits continue to drive willingness to disclose information to a technological device, even a technology developed for community benefits. The dyadic perspective helped us explore the influence parents have on teens and vice versa. Future research could leverage this implementation of the calculus to study other dyads with power relationships (e.g., employee-manager, doctor-patient) or dyads with an expectation of more egalitarian relationships (e.g., roommates, spouses, adult siblings).

It is important to note in our discussion that, as stated in the beginning of the paper, the use of CT apps was voluntary in many countries but mandatory in others. In this study, participants were all from the US, where CT app use was largely voluntary (some individuals might have been obligated to use a form of CT apps for their work environment). Therefore, our results mainly reflect the volitional nature of CT app usage. Furthermore, they may not be generalizable to other geographical areas such as Europe where privacy laws are substantially different (i.e., GDPR). Anecdotal data reported in the first paragraph of this paper suggest that mandatoriness does significantly help in the diffusion of such community technologies. As governments and organizations consider diffusion of other community technologies, it will be important for researchers to further examine the effects of mandatoriness. For example, the university of one of the authors announced in June 2021 that students must be vaccinated to be enrolled in the university in August 
2021 (as did several other universities). The university later announced that it was also mandatory for all staff and faculty to be vaccinated. Will vaccination passports also become mandatory? How do individuals feel about their privacy with such passports? How will families negotiate use of passports? Will all members have to use a passport? If not, can they travel together?

Finally, the study also has important implications for the design of the artifact, the contract tracing app, itself. Vinuesa, Theodorou, Battaglini and Dignum [43] suggest that CT apps need more transparency and accessibility and require users to be better informed and capable of using the apps correctly. Our results further support these ideas since it is apparent that teens, and likely parents as well, did not clearly understand how CT apps really worked. Much work is needed to explore these possibilities.

\section{Conclusion}

Our study of intergenerational family dyads' use of CT apps offered fresh insights into a unique type of interactive unit, with both anticipated and novel results. Because each family's identity may extend beyond their immediate household, it is likely that that this domino effect will increase adoption outside of the household to their extended family and broader social networks. There is also the need to study other 'tightknit' groups to see how their perceptions, intentions and behaviors affect each other's.

In this paper, we applied and extended the privacy calculus to parent-teen dyads longitudinally and found that the privacy calculus held true with some variations in cross-actor effects. This finding presents an array of new possibilities for the application and study of information privacy, and for avenues of interdisciplinary work with various populations, such as families, couples, and co-workers.

\section{Acknowledgements}

This research was supported in part by a grant from the Institute for Society, Culture, and the Environment at Virginia Tech, and by the National Science Foundation (SES- 2039172).

\section{References}

[1] Servick, K. COVID-19 contact tracing apps are coming to a phone near you. How will we know whether they work? , City, 2020.

[2] Altshuler, T. S. and Hershkovitz, R. A. Digital Contact Tracing and the Coronavirus: Israeli and Comparative Perspectives. Foreign Policy at Brookings (2020).
[3] Hsu, J. Contact Tracing Apps Struggle to Be Both Effective and Private. City, 2020.

[4] VDH Why Should You Download COVIDWISE? , City, 2021.

[5] Chan, E. Y. and Saqib, N. U. Privacy concerns can explain unwillingness to download and use contact tracing apps when COVID-19 concerns are high. Computers in Human Behavior, 119, 106718 (2021), 1-12.

[6] Compton, L. D. and Keller, B. A. The ongoing US vaccine passport debate. City, 2021.

[7] Whitney, L. Most Americans say 'No' to coronavirus contact tracing apps. City, 2020.

[8] Allen, K. R. and Henderson, A. C. Family theories: foundations and applications. Wiley Blackwell, West Sussex; Malden, MA, 2017.

[9] Bronfenbrenner, U. Ecology of the family as a context for human development: Research prospectives. Developmental Psychology, 22, 6 (1986), 723-742.

[10] Buehler, C. Family Processes and Children's and Adolescents' Well-Being. Journal of Marriage and Family, 82, 1 (2020), 145-174.

[11] Dworkin, J. Adolescents as the Family Technology Innovators. Springer, City, 2018.

[12] Dworkin, J., Rudi, J. H. and Hessel, H. The state of family research and social media. Journal of Family Theory \& Review, 10, 4 (2018), 796-813.

[13] Katz, V. S., Moran, M. B. and Gonzalez, C. Connecting with technology in lower-income US families. New Media \& Society, 20, 7 (2018), 2509-2533.

[14] Dworkin, J., Hessel, H. and LeBouef, S. The Use of Communication Technology in the Context of Adolescent and Family Development: An Integration of Family and Media Theories. Journal of Family Theory \& Review, 11 (2019/11/01 2019).

[15] Schoppe-Sullivan, S. J. and Fagan, J. The Evolution of Fathering Research in the 21st Century: Persistent Challenges, New Directions. Journal of Marriage and Family, 82 (2020), 175-197.

[16] Dinev, T. and Hart, P. An Extended Privacy Calculus Model for E-Commerce Transactions. Information Systems Research, 17, 1 (2006), 61-80.

[17] Jiang, Z., Heng, C. S. and Choi, B. C. Privacy Concerns and Privacy-Protective Behavior in Synchronous Online Social Interactions. Information Systems Research, 24, 3 (2013), 579-595.

[18] Laufer, R. S. and Wolfe, M. Privacy as a concept and a social issue: A multidimensional developmental theory. Journal of Social Issues, 33, 3 (1977), 22-42.

[19] Bélanger, F. and James, T. L. A Theory of Multilevel Information Privacy Management for the Digital Era. Information Systems Research, Articles in Advance (2020), 1-27.

[20] Bélanger, F., Crossler, R. E. and Correia, J. Privacy Maintenance in Self-Digitization: The Effect of Information Disclosure on Continuance Intentions. The Datbase for Advances in Information Systems, 52, 2 (2021 ), 7-24.

[21] Anderson, C. L. and Agarwal, R. The Digitization of Healthcare: Boundary Risks, Emotion, and Consumer Willingness to Disclose Personal Health Information. Information Systems Research, 22, 3 (2011), 469-490. 
[22] Krasnova, H., Spiekermann, S., Koroleva, K. and Hildebrand, T. Online social networks: why we disclose. Journal of Information Technology, 25, 2 (2010), 109-125. [23] Bélanger, F. and $\mathrm{Xu}, \mathrm{H}$. The Role of Information Systems Research in Shaping the Future of Information Privacy. Information Systems Journal, 26, 6 (2015), 573578.

[24] Bélanger, F. and Crossler, R. E. Privacy in the digital age: A review of information privacy research in information systems. MIS Quarterly, 35, 4 (2011), 1017-1042.

[25] Mollborn, S., Fomby, P., Goode, J. A. and Modile, A. A life course framework for understanding digital technology use in the transition to adulthood. Advances in Life Course Research, 47, 100379 (2020), 1-12.

[26] Steinberg, L. Age of Opportunity: Lessons from the new science of adolescence. Houghton Mifflin Harcourt, New York, NY, 2015.

[27] Walker, S. K. Technology use and families: Implications for work-family balance and parenting education. . United Nations Department of Economic and Social Affairs (UNDESA) Division for Inclusive Social Development. , 2021.

[28] Bélanger, F., Crossler, R. E., Hiller, J. S., Hsiao, M. and Park, J.-P. POCKET: A Tool for Protecting Children's Privacy Online. Decision Support Systems, 54, 2 (2013), 1161-1173.

[29] Smith, H. J., Dinev, T. and Xu, H. Information privacy research: An interdisciplinary review. MIS Quarterly, 35, 4 (2011), 989-1016.

[30] Debatin, B., Lovejoy, J. P., Horn, A. K. and Hughes, B. N. Facebook and online privacy: Attitudes, behaviors, and unintended consequences. Journal of Computer-Mediated Communication, 15, 1 (2009), 83-108.

[31] Koohikamali, M., Peak, D. A. and Prybutok, V. Beyond Self-Disclosure: Disclosure of Information about Others in Social Network Sites. Computers in Human Behavior, 69 (2016), 29-42.

[32] Bélanger, F. and Crossler, R. E. Dealing with digital traces: Understanding protective behaviors on mobile devices. The Journal of Strategic Information Systems, 28 (2018/11/23/2019), 34-49.

[33] Hiller, J., Belanger, F., Hsiao, M. and Park, J. M. POCKET protection. American Business Law Journal, 45, 3 (2008), 417-453.

[34] Rosseel, Y. lavaan: An R Package for Structural Equation Modeling. Journal of Statistical Software, , 48, 2 (2012), 1-36.

[35] Kashy, D. A. and Kenny, D. A. The analysis of data from dyads and groups. Cambridge University Press, City, 1999).

[36] Blackwell, L., Gardiner, E. and Schoenebeck, S. Managing Expectations: Technology Tensions among Parents and Teens. City, 2016.

[37] Clark, L. S. How Parents Are Mediating the Media in Middle-Class and Less Advantaged Homes. Oxford Scholarship City, 2013.

[38] De La Garza, A. Contact tracing apps were big tech's best idea for fighting COVID-19. Why haven't they helped? TIME, City, 2020.

[39] Lehmann, C. Privacy concerns hindering digital contact tracing. City, 2020.
[40] Acquisti, A. Privacy in Electronic Commerce and the Economics of Immediate Gratification. City, 2004.

[41] Bélanger, F. and Crossler, R. E. Privacy in the Digital Age: A Review of Information Privacy Research in Information Systems. MIS Quarterly, 35, 4 (2011), 10171041.

[42] Sheeran, P. Intention-Behavior Relations: A Conceptual and Empirical Review. European Review of Social Psychology, 12, 1 (2002), 1-36.

[43] Vinuesa, R., Theodorou, A., Battaglini, M. and Dignum, V. A socio-technical framework for digital contact tracing. Results in Engineering 8, 100163 (2020), 1-4. 